\title{
IL POTERE DI JUDIT
}

\section{MONICA BILOTTA}

L'ambivalente Giuditta veterotestamentaria trova una delle sue incarnazioni letterarie più affascinanti nella Judit di Federico Della Valle, tragedia in cui la figura biblica si trasforma in un'inedita eroina controriformistica, ossimorica vergine seduttiva e paladina della fede, capace di un'incisiva azione politica ${ }^{1}$. In questo dramma, la riflessione sui rapporti di forza all'interno delle corti, tipica dell'opera del tragediografo astigiano, si coniuga con un'innovativa rappresentazione delle relazioni di potere tra $\mathrm{i}$ sessi ${ }^{2}$. Così come nell'Ester - anch'essa di argomento biblico —, nella Judit il personaggio eponimo si trova ad affrontare un avversario maschile ${ }^{3}$. La dinamica dello scontro è analoga nei due testi: in entrambi i casi la donna è legata al comandante o al sovrano da un vincolo erotico, sconfigge il servo-consigliere e raggiunge il suo scopo grazie alla capacità di manipolare a proprio vantaggio le attese degli interlocutori.

Nei due drammi di Della Valle la conquista del "cuore" del capo supremo rappresenta per tutti i subordinati l'unica mediazione possibile per accedere al potere politico ${ }^{4}$. La problematica possibilità di un'azione che

${ }^{1}$ Sulle complesse valenze del personaggio di Judith nel testo biblico e sull'utilizzo contrapposto fattone dalla Riforma Protestante e dalla Controriforma Cattolica cfr. Stocker, Judit Sexual Warrior. Vorrei esprimere la mia profonda gratitudine a Laura Sanguineti White e ad Andrea Baldi, del dipartimento d'Italiano di Rutgers, per l'incoraggiamento e il sostegno offertomi nelle ricerche su questo soggetto.

2 Il ruolo centrale assunto dalla rappresentazione del potere nelle tre tragedie è stato ripetutamente messo in luce, ma mai studiato nella sua specificità.

${ }^{3}$ Si tratta di Aman nell' Ester e di Vagao nella Judit. A conferma della regolarità di questa struttura, si pensi nell' Ester alla lotta di Zares e Dagan per il cuore di Aman, scontro che riproduce, a un livello sociale inferiore, quello tra Ester ed Aman per il cuore di Assuero. Alla dinamica che contrappone le figure dei consiglieri nelle tragedie dellavalliane allude Sanguineti White, Dal detto alla figura, 73.

${ }^{4}$ Lo scontro, che in entrambe le tragedie oppone l'eroina al consigliere fraudolento e i servi fedeli ai servi malvagi, ha sempre per oggetto le emozioni e le passioni del sovrano. La possibilità per i subordinati di acquisire potere e di intervenire nella storia passa letteralmente attraverso il "cuore" del proprio superiore. Non a caso, nel momento culminante della loro esaltazione, tanto Vagao nella Judit quanto Aman nell' Ester si gloriano di essere "spirto", "cuore" ed "alma" del proprio signore (Cfr. Judit, vv. 522-524; Ester, vv. 742-750; v. 759). 
incida sul reale si lega così, secondo un canone tipico dell'età barocca, alla questione del controllo delle coscienze. La figura di Judit esempla in maniera particolarmente riuscita i dilemmi posti dal complesso rapporto fra la dimensione etica dell'agire umano e la sua efficacia concreta.

Eroina della dissimulazione, Judit non ricorre mai alla menzogna con i suoi avversari, si limita a tacere, a non smentire le loro ipotesi, operando al tempo stesso in nome di Dio5. Per l'appunto, non simula, dissimula. Della Valle, dal suo osservatorio di piccolo funzionario soggetto alla ruota della fortuna, assolve e giustifica l'utilizzo dell'unico strumento a disposizione dei sottoposti per conservare un margine d'intervento autonomo nei confronti tanto del potere politico quanto della Chiesa. Di fronte ai personaggi femminili, l'autore mostra un'apertura simile a quella che rivela riguardo alla dissimulazione, conferendo legittimità etica all'utilizzo di strumenti di autodeterminazione censurati dalla tradizione quali il mascheramento dei propri obiettivi e l'esaltazione della propria attrattiva sensuale.

Sia nella Judit che nell'Ester la donna è relegata dalle convenzioni sociali in una posizione secondaria; costituisce un elemento decorativo e un oggetto del desiderio maschile, che la vorrebbe, tutt'al più, specchio della potenza del consorte. Tuttavia, le protagoniste dellavalliane rivendicano, sotterraneamente, un proprio margine di azione e sono in grado di incidere con efficacia sul reale grazie alla capacità di decostruire dall'interno i preconcetti degli uomini, che, pur facendole soggetto del proprio sguardo, sono incapaci di vederle davvero. Affiora così, seppure in forma obliqua, il conflitto tra i sessi. La consapevolezza con cui i personaggi femminili fingono di adeguarsi al pregiudizio maschile, costruendosi scientemente come

${ }^{5}$ La dissimulazione onesta delle due figure bibliche e in particolar modo quella di Judit, costretta a muoversi in campo avverso, ricorda il comportamento delle eroine tassiane della simulazione: Sofronia e Armida. In Tasso l'opposta valutazione delle due giovani donne si basa sull'antitesi dei fini perseguiti ed esula da ogni considerazione intorno al mezzo adottato. Nelle tragedie di Della Valle emerge la stessa priorità del fine edificante sul mezzo moralmente discutibile. Rispetto a Tasso, però, il tragediografo astigiano accentua ulteriormente l'ambiguità di comportamento della propria protagonista. A differenza di Sofronia, che si limita a celare ad Aladino il suo vero obiettivo in nome di una giusta causa, senza curare la seduttività del proprio aspetto, Judit usa consapevolmente il suo fascino per oscurare la capacità di giudizio degli Assiri, comportandosi come Armida fra i príncipi cristiani. Poiché i testi di Della Valle costituiscono un'appassionata riflessione sulla realtà cortigiana, l'assoluzione dell'ambivalenza del comportamento di Judit si riverbera su quel circolo di relazioni, in modo non previsto nel poema tassiano. 
immagini al tempo stesso inermi e sensuali, viene integralmente giustificata dal poeta sin dalla dedica, in cui Judit ed Ester vengono proclamate figure della Vergine ${ }^{6}$. Il ricorso al richiamo dell'eros viene, dunque, assolto e valorizzato come strumento legittimo di un'azione politica voluta da Dio, in contrasto con le crescenti limitazioni poste all'agire delle donne in età controriformista e con la relativa condanna di immoralità per ogni loro iniziativa estranea alle cure domestiche?

Tale apertura nei confronti del femminile costituisce un tratto uniformemente presente nella produzione del drammaturgo astigiano. Un primo dato risalta già dai titoli delle sue tragedie (Judit, Ester, La Reina di Scozia) e della sua tragicommedia (Adelonda di Frigia), opere tutte incentrate su protagoniste illustri. Inoltre, le sue figure di donna svolgono ruoli sociali diversi (al di là della preminenza di due protagoniste regine), senza che i loro vizi e le loro virtù siano vincolate ad un modello uniforme: possono essere timide come Abra, ambiziose come Zares, ispirate e coraggiose come Judit, spietate come Isabella. Nessun pregiudizio ideologico apologetico o denigratorio, dunque, ma nemmeno cecità di fronte alle peculiarità del loro sesso, tanto che ad essere in gioco è proprio lo statuto della donna in quanto oggetto e l'implicita aspirazione a una nuova soggettività.

Della Valle ha ben presente la percezione sociale del ruolo, del comportamento e persino del corpo della donna. Il suo sguardo è acuto e atten-

${ }^{6}$ Nella "Dedica della Iudit e dell' Ester secondo l'edizione a stampa" il drammaturgo invoca la regina dei cieli chiedendole: "Furo tue ombre queste donne [...] permetti che Iudit ed Ester, se fûr già dette tue figure, or si dicano anche cose tue" (Della Valle, Tragedie, 55). Judit, in particolare, viene evocata nel prologo in termini di suprema bellezza: "immagine bella / d'altra di te più bella, ancor non nata" (vv. 41-42). A questa edizione si farà d'ora innanzi riferimento con la semplice indicazione dei versi citati. Lorigine controriformista della lettura di Judit quale figura della Vergine e le imprevedibili conseguenze di tale accostamento vengono sondate nel saggio di Pietropaolo, "Iudit, Femme Fatale of the Baroque Stage." Elena Ciletti ricostruisce le radici altomedievali dell'interpretazione figurale del personaggio di Judit e si sofferma sulla coesistenza tardorinascimentale di una duplice iconografia dell'eroina biblica, percepita sia come mulier fortis e virago sia come archetipo di femminilità lussuriosa e distruttiva nel suo "Patriarchal Ideology in the Renaissance Iconography of Judith."

${ }^{7}$ Si pensi allo storico saggio di Joan Kelly, "Did Women Have a Renaissance?" I testi dellavalliani si riallacciano al dibattito cinquecentesco fra detrattori e celebratori della donna, ponendosi tra le poche voci esplicitamente a favore del "sesso donnesco". Sulla controversia si vedano Chemello, "Donna di palazzo"; Daenens, "Superiore perchè inferiore"; King, Women of the Renaissance, Benson, The Invention of the Renaissance Woman. 
to; l'originalità del suo atteggiamento lo potrebbe far ritenere persino un po' complice, tanto da fargli rovesciare e decostruire il profilo convenzionale della comprimaria paurosa e imbelle, vista quale figura del desiderio, ma anche come elemento decorativo, strumento di rappresentanza. L'uno è il destino riservato a Judit da Oloferne, e ancor più da Vagao, l'altro il modo in cui Aman guarda Ester: entrambe le eroine sono coscienti di essere soggette a questo filtro deformante nello sguardo dei deuteragonisti. La novità della soluzione di Federico Della Valle consiste nell'aver messo in scena la consapevolezza, da parte delle donne, del pregiudizio da cui è affetto l'occhio maschile e nell'aver attribuito alle sue protagoniste un uso strumentale di tale cognizione. La bellezza risulta l'arma fondamentale delle due figure bibliche ${ }^{8}$, insieme, in particolar modo nel caso di Judit, al malinteso senso di superiorità maschile, tale da far credere che l'Ebrea di stirpe guerriera sia, come ella stessa dichiara, "vil donna, commossa da ogni fronda mossa" (vv. 19881989). La capacità femminile di valersi del pregiudizio maschile rappresenta la specifica marca di genere riconosciuta da Federico Della Valle alle sue protagoniste. La descrizione della bellezza delle eroine, l'attenzione al loro abbigliarsi e le scene di seduzione sono da ricondurre a quest'ottica, prima che ad una specifica intenzione edonistica o voyeuristica dell'autore. In questo egli si discosta tanto dalle contemporanee scelte di Marino quanto dalla turbata attrazione di Tasso verso la sensualità femminile.

Come si è osservato, le figure femminili dellavalliane sono parte integrale del sistema di forze che regola le tragedie. Così, Ester e Judit condividono, con i dovuti distinguo, la condizione di regina, di compagna del re/condottiero?. In tale veste, il loro ruolo istituzionale pare soprattutto decorativo. Judit, poco meno che prigioniera di guerra, è ovviamente subordinata ad Oloferne, e la stessa Ester, regina a pieno titolo, è sottomessa al marito, né può intervenire direttamente per salvare il proprio popolo. Tuttavia, il suo invito ad Aman rappresenta per lui un'ascesa in termini di prestigio e di potere: la regina trasmette il potere senza goderne le prerogative, essendo di per sé dotata solo del lustro che compete alla consorte del sovrano ${ }^{10}$.

${ }^{8}$ Scrive Trombatore: "la bellezza è l'unica insidiosa arma di Giuditta" ("Le tragedie di Federico Della Valle" 174).

${ }^{9}$ Se Ester è regina di Persia al fianco di Assuero, Oloferne auspica che Judit accetti di diventare sua consorte. Nel cuore di Oloferne, che riveste in scena il ruolo di figura di Nabucodonosor, sovrano d'Assiria, Judit è, dunque, a propria volta "reina" (cfr. v. 1845 e v. 1890).

${ }^{10}$ Le due regine della terza tragedia, Isabella e Maria, sono invece delle vere sovrane, le cui caratteristiche saranno da esaminare insieme a quelle degli altri monarchi delle tragedie di Della Valle. Si può anticipare sin d'ora che la connotazione di gender influenza solo in maniera marginale la costruzione di questi personaggi e la loro connotazione morale. 
Pur rivestendo ufficialmente un ruolo subordinato, le due eroine esercitano però, nei confronti del loro compagno e re, un influsso che permette loro di agire autonomamente e di raggiungere i propri scopi. E il potere dato loro dalla bellezza, che seduce e innamora, legando il volere dell'uomo ai loro disegni. Come avviene nel rapporto re/consigliere, nel vincolo tra re e regina il potere non si concentra nelle mani di uno solo degli interlocutori. Ad essere precisi, anzi, in entrambe le tragedie è il potere del sovrano sulla consorte a mostrarsi più apparente che reale: Assuero, vinto dal fascino della moglie, rinuncia alla facoltà, conferitagli dalla legge, di condannarla a morte; Oloferne, nell'invitare Judit al suo tavolo e nel suo letto, si mette in realtà nelle mani di lei.

Ester e Judit si servono entrambe dell'arsenale della femminilità curando le proprie vesti e la propria gestualità e, nel far questo, nascondono i loro veri propositi. Tuttavia, mentre la dissimulazione di Ester è di breve durata, limitandosi alle due cene col consorte e Aman, quella di Judit si estende a tutto il testo. Differente è, d'altro canto, la situazione delle due eroine: ambedue rischiano la rovina della propria gente, ma, mentre la regina di Persia combatte la propria battaglia su un territorio ben conosciuto, Judit è in campo nemico ed il suo proposito è ben più impegnativo e sanguinario. La protagonista tace a tutti i suoi progetti, persino alla serva Abra, alla quale pure mostra un animo coraggioso ed ispirato, che confida nella grazia e nella misericordia divina. La sua continua dissimulazione ed il suo statuto incerto all'interno del campo assiro rendono Judit un personaggio più elusivo e complesso di Ester, e che meglio incarna un modello di femminilità trionfante.

La cifra del personaggio di Judit è il suo enigmatico sorriso. L'eroina ebrea lascia agli interlocutori il compito di interpretare i suoi pensieri, senza svelarsi, a differenza del verboso Oloferne, che indulge ad una continua, e forse compiaciuta, autoanalisi ${ }^{11}$. Non abbiamo una descrizione caratterizzante di Judit; la sua avvenenza, ripetutamente menzionata e celebrata, resta priva di connotati precisi. Di lei conosciamo solo il colore dora-

${ }^{11} \mathrm{Si}$ veda in proposito l'analisi di Sanguineti White, Dal detto alla figura, 112. La rappresentazione della loquacità del personaggio maschile e del silente autocontrollo del personaggio femminile capovolge gli attributi tradizionali dei due sessi, destinati ad essere riconfermati proprio in merito alla dissimulazione nel trattato di Torquato Accetto, Della dissimulazione onesta, di pochi decenni posteriore alle tragedie dellavalliane. In questo sintetico saggio le lacrime di Penelope, incapace di controllare - come tutte le donne - la manifestazione delle proprie passioni, vengono contrapposte alla "fermezza del corno e del ferro" di Ulisse, virilmente capace del più completo autocontrollo (Della dissimulazione onesta, 29). 
to dei capelli, il candore delle carni e, per l'appunto, il sorriso. Filtrata dalle percezioni altrui, avvolta da un susseguirsi di interpretazioni soggettive, Judit risulta ancora più misteriosa e sfuggente di quanto non la rendano le sue parole, miranti intenzionalmente a lusingare l'interlocutore e le sue attese.

Sotto le vesti di donna imbelle, timorosa e compiacente, combatte la propria guerra per la salvezza della patria, facendo leva, scientemente, sulle aspettative maschili. Vagao vede in lei un'umile prigioniera, desiderabile agli occhi del condottiero e dunque capace di guadagnare al servo-consigliere un insperato controllo sul proprio capo. Judit sembra agire in sintonia con queste percezioni, dicendosi umile compagna di schiavitù, lusingata dall'amicizia del mezzano. Per Oloferne Judit è l'oggetto del desiderio erotico non meno che sentimentale, e rappresenta l'occasione attesa per un'avventura distensiva in mezzo alle azioni militari; né le parole di Judit sembrano contraddirlo ${ }^{12}$. Per il fedele Arimaspe è una sirena allettatrice, capace, pur senza volerlo, di allontanare il comandante dai suoi doveri bellici13. La straordinaria bellezza di Judit, però, smantella persino le sue motivate riserve, inducendolo ad approvare le scelte di Oloferne ${ }^{14}$.

Per mettere a fuoco le camaleontiche virtù di Judit e le strategie con cui si conforma alle aspettative maschili per manipolarle, occorre soffermarsi sulla sua celebre toletta, narrata da Vagao ad Oloferne. Si tratta di un episodio centrale per importanza e incidenza strutturale, dove alle lusinghe dei sensi si somma la fascinazione della parola, capace di legare e avvincere. Oloferne ha incaricato Vagao di recarsi da Judit e di metterle il suo tesoro a disposizione; nell'obbedire, il servo ha sorpreso l'eroina nell'atto di svestirsi e si è voyeuristicamente fermato a spiarla. Nel punto culminante del dramma, il servo riferisce al suo comandante ciò che ha visto, per sfruttare il potere che gli viene dalla posizione di intermediario.

Il ruolo chiave rivestito dalla parola rende questa scena di seduzione particolarmente suggestiva dal punto di vista metatestuale. È possibile infatti riconoscere in questi versi una riflessione sul potere persuasivo della parola e dell'immagine, elementi costitutivi di ogni opera teatrale.

\footnotetext{
${ }^{12}$ Fra i molti possibili esempi si veda, in particolar modo, la risposta di Oloferne alle pressioni di Arimaspe, ai vv. 1178 - 1201.

13 Arimaspe vede nell'avventura erotica cui il comandante indulge, proprio nel momento culminante dell'assedio, un possibile segno di corruzione del valore guerresco dell'esercito assiro (vv. 1044-1053). Il pericolo non sarebbe, dunque, rappresentato da Judit in sé, quanto dall'attenuarsi dello sforzo bellico.

${ }^{14}$ Per un diverso giudizio sulla ricezione di Judit tramite gli epiteti usati dai suoi interlocutori si veda Sanguineti White, Dal detto alla figura, 92-96.
} 
Nello svolgere questa operazione, Della Valle approfitta di un momento istituzionale della tragedia, genere cui Aristotele aveva riservato la mimesis, non la diegesis. Ad ospitare l'analisi dei rapporti tra parola e immagine è, infatti, la rhesis del messaggero, momento deputato al racconto di ciò che, per motivi rituali, non poteva avere luogo in scena, e dunque unico spazio potenzialmente dedicato all'ekphrasis all'interno del dramma. Nel trasformare la rhesis in ekphrasis Della Valle aggiunge un ulteriore livello al proprio intervento metatestuale.

La descrizione della toletta di Judit, infatti, permette di mettere in scena e commentare (tramite gli enunciati del coro) il modo in cui la parola e l'immagine sono in grado di plasmare le reazioni emotive e di condizionare le abilità raziocinanti dell'uomo ${ }^{15}$. Della Valle esplora, in particolare, lo statuto della tragedia e, in generale, le modalità e la dimensione etica di ogni operazione persuasiva fondata sulla percezione visiva ed auditiva. In tale contesto Oloferne diventa figura sia dello spettatore, che si abbandona consapevolmente al fascino dell'opera teatrale, abdicando alla capacità di valutare criticamente il messaggio, sia di ogni individuo sedotto da un discorso sapiente che sfrutti il richiamo dell'eros. Lautore intreccia questa indagine conoscitiva ad un'analisi sui rapporti tra parola e immagine, condotta facendo continuo ricorso al campo metaforico della visione come pittura interiore, operazione che incoraggia la lettura della scena della toletta di Judit quale intenzionale presa di posizione all'interno del dibattito sui rapporti tra poesia e pittura.

Attraverso la sua dettagliata descrizione dello svestirsi e del successivo abbigliarsi dell'eroina, Vagao lusinga la fantasia del padrone sulle gioie dell'eros che questi si attende e ne accarezza il desiderio, rispondendo appieno all'invito di Oloferne che lo esorta ad un resoconto minuzioso:

Tutto di', nulla lascia.

Comincerò, ascoltando,

il ben ch'avrò abbracciando.

Oloferne conta, dunque, di godere, grazie alle parole del servo, un'anticipazione del possesso di Judit. La ricezione del racconto prepara il con-

${ }^{15} \mathrm{La}$ dimensione puramente immaginativa e verbale della seduzione di Oloferne viene sottolineata da Angelini (Il teatro barocco, 174) e da Getto ("Il teatro barocco di Federico Della Valle," 1496-1498). Si veda inoltre la suggestiva analisi di Tessari ("La Iudit di Della Valle," 109-124). Tessari rilegge la natura obliqua della seduzione di Oloferne entro il "dramma cosmico" che a suo avviso struttura l'intera tragedia. La forza d'attrazione della bellezza, possibile strada di accesso a Dio, diviene fonte di labirintico smarrimento per il principio maschile rappresentato dal comandante assiro. 
tatto fisico, come sottolinea la rima baciata "ascoltando" : "abbracciando". La parola prefigura il piacere dei sensi, in quanto è in grado di creare un'immagine efficace nella mente dell'ascoltatore: ne consegue che la poesia, per conquistare le emozioni di chi ne fruisce, deve ricorrere agli strumenti della pittura.

Questo motivo viene sviluppato nel successivo intervento di Oloferne, che, interrompendo la narrazione del servo, esclama:

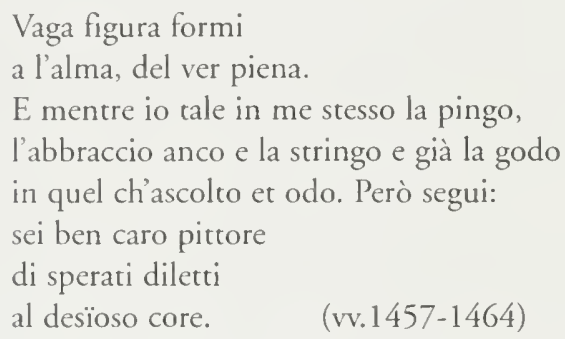

Vagao viene definito "pittore" poiché il suo racconto offre una rappresentazione visuale alla facoltà immaginativa di Oloferne; ascoltando, Oloferne a sua volta "dipinge" nel proprio intimo Judit ed ha così l'illusione di goderne le grazie. Viene qui rapidamente delineata una vera e propria teoria della ricezione: nel restituire una scena visiva, la parola, con la sua valenza icastica, crea un'immagine mentale che predetermina le reazioni emotive dell'ascoltatore ${ }^{16}$. L'implicita comparazione tra Vagao che con la sua descrizione diventa controfigura del poeta-autore - e un pittore pone in risalto l'intercambiabilità delle due arti (poesia e pittura) e l'analogia dei loro effetti sull'ascoltatore/spettatore. Della Valle sembra dunque riprodurre il binomio oraziano dell'ut pictura poësis. la poesia equivale alla pittura, in quanto suggerisce un'immagine alla mente dell'ascoltatore e ne influenza lo stato emotivo.

Oloferne sembra ravvisare l'indole manipolatrice del servo nel momento in cui lo definisce "pittore / di sperati diletti / al desïoso core"; pur avvertendo le lusinghe dell' ars narrandi di Vagao, non riesce, però, a compiere il passo successivo e a sospettare, se non smascherare, gli scopi del

${ }^{16}$ Si stabilisce qui un suggestivo parallelo tra visione e possesso e tra ascolto e godimento, come testimoniano le rime interne: "pingo"/ "stringo", "godo"/ "odo". La disposizione chiastica fra i due verbi di percezione ("in me stesso la pingo, / ... odo"), posti all'esterno, e i due verbi di 'fruizione, collocati all'interno, sottolinea sia l'opposizione tra il presente e la proiezione futura, sia il contrasto tra la natura immateriale della percezione e la fisicità del possesso, peraltro solo immaginato. 
mezzano. Il capo assiro mostra un’analoga cecità nei confronti di Judit, di cui riconosce il carattere guerriero, senza però intuirne il secondo fine. A differenza dei suoi interlocutori, Oloferne non sa misurare il potere della parola come arma di offesa e di difesa, ma rimane prigioniero di una concezione materialistica ed elementare dei conflitti.

Tale consapevolezza affiora invece nei commenti del coro, che, a conclusione del racconto di Vagao, approfondisce l'analisi dei rapporti tra parola e immagine segnalando le differenze tra i due strumenti e decretando il trionfo della resa verbale, ovvero della poesia:

O di servo Vagao voci ben degne,

voci finte, depinte

di lusinghier diletto,

che con le voci serpe

a frastornar il petto!

Tutto può vista vaga.

Smuove, travolve, accende,

e contra lei un cuor mal si difende.

Ma lingua, che dipinge

a cor già acceso placida figura,

ahi, quanto lega e stringe! (vv. 1611-1621).

Oltre ad essere a tutti gli effetti un'entità drammatica che interagisce con i personaggi, il coro dei soldati assiri assolve anche la funzione di pronunciare un giudizio etico sulle vicende che si svolgono in scena ${ }^{17}$. In questo passo, il coro si sofferma sugli effetti della parola e dell'immagine, insistendo sull'uso manipolatorio a cui questi mezzi possono essere piegati. In particolare, il potenziale della parola nel suggestionare la mente dell'ascoltatore è visto conve superiore a quello della visione. Si tratta di una questione centrale nell'epoca della Controriforma, poiché parola e immagine sono gli strumenti adoperati dallo Stato e dalla Chiesa per assicurarsi il controllo delle coscienze. Della Valle, dunque, non solo interviene nel secolare dibattito sul rapporto tra pittura e poesia, ma assegna alla propria indagine un valore conoscitivo ed etico in sintonia col tema fondamentalmente politico delle sue tragedie.

Le parole di Vagao vengono definite "finte", ossia 'disposte con artificio', "depinte/ di lusinghier diletto", cioè 'arricchite (ma la scelta del verbo

${ }^{17}$ Riguardo al duplice ruolo del coro, che accomuna la tragedia cinquecentesca alla drammaturgia classica, si vedano le osservazioni di Paratore in "Nuove prospettive sull'influsso del teatro classico nel '500" e La Penna in "Palazzo, coro e popolo nella tragedia antica e nella tragedia umanistica." 
è significativa) di un piacere che intende allettare'; piacere che, attraverso l'esca verbale, procede obliquo, insidioso ("serpe"), fino al cuore ("petto") per irretirlo. La censura morale del coro assume, quindi, valore di ammonimento per gli spettatori, sottoposti, nel quotidiano, a sollecitazioni simili. $\mathrm{Al}$ tempo stesso, in un'ulteriore torsione cognitiva, la tragedia cerca, a sua volta, di 'sedurli' proprio nel momento in cui svela i meccanismi della persuasione. La parola che lusinga l'ascoltatore è quella che diletta, che asseconda i suoi desideri; compiacendone le attese, essa risulta di per sé pericolosa, ambigua, potenzialmente manipolatrice (non a caso, le parole di Vagao si addicono ad un'anima servile, come rimarca il v. 1611).

Dopo questa premessa sull'insidia di un discorso congegnato ad arte, inteso a soggiogare il destinatario, il coro passa ad una notazione sul potere dell'immagine e della parola: una bella immagine ("vista vaga") ha un effetto potente, pervasivo, su chi la contempla ("tutto può"/ "contra lei un cor mal si difende"); agisce sulle emozioni dello spettatore, che domina e inframma. Tuttavia, la trascrizione verbale di un'immagine allettante, ad opera di una "lingua che dipinge", è ancora più forte nel legare un "cor già acceso", come sottolinea l'avversativa "ma".

Se alla rappresentazione visiva, e dunque alla pittura, spetta, in primo luogo, la capacità di suscitare un'intensa risposta emotiva, la parola è in grado di ricreare e rendere stabile l'impressione così generata ${ }^{18}$. In virtù del potere catalizzante della resa verbale, l'oratore può indirizzare ai propri fini le emozioni destate dalla visione interiore che ha saputo evocare nell'ascoltatore. Se entrambe le arti, poesia e pittura, hanno la medesima forza nel sollecitare risposte emotive, l'autore suggerisce, per bocca del coro, che

18 L'importanza assegnata alla capacità di creare, tramite la parola, un'immagine nella mente degli ascoltatori viene teorizzata dalla retorica gesuitica durante la prima metà del '600, ossia proprio nel periodo della composizione della Judit o in anni immediatamente successivi. Se La Reina di Scozia è stata definita, a ragione, da Mercuri, una tragedia gesuitica ("La Reina di Scozia di Federico Della Valle," 147 e seg.), non sorprenderà che si possano riscontrare varie analogie tra Della Valle e l'estetica gesuitica, affinità che non impedisce al tragediografo di trarre le proprie autonome conclusioni morali ed estetiche. Un elemento di differenziazione è costituito, ad esempio, dal forte ambito erotico in cui è inserita la riflessione dellavalliana sul potere della parola e dell'immagine. Sull'importanza della componente visiva, esteriore e interiore, nella poetica gesuitica si vedano, tra gli altri, Franca Angelini, che ricorda come, a partire dagli Esercizi spirtuali di Ignazio de Loyola, la Controriforma privilegi "l'oratoria e le arti figurative, che concorrono a "muovere più l'affetto et intenerire il cuore" ("Poesia e letteratura tragica," 203) e Marc Fumaroli, Eroi e oratori, soprattutto alle pp. 198-211, dedicate alla produzione del gesuita Bernardino Stefonio. 
la parola si presta meglio dell'immagine ad un uso strumentale da parte del potere.

Il primato della parola viene sancito dal ritratto - in versi - che Vagao delinea per il proprio signore. Si tratta di uno dei passi più riusciti dell'intera tragedia: la descrizione della toletta di Judit si prolunga per poco meno di duecento versi (vv. 1420-1605), in cui l'autore allestisce una scena dinamica e insieme ricca di dettagli. La rhesis del mezzano si trasforma in ekphrasis, descrivendo un vero e proprio quadro, anzi un dittico, in quanto comprende anche la tavola elaborata dall'eroina. Dopo avere scoperto la presenza del voyeur all'ingresso della sua tenda, Judit, infatti, lo invita ad entrare ed assistere a quel che resta delle sue abluzioni e diviene, così, da oggetto inerme di uno sguardo indiscreto regista consapevole della propria bellezza. È questo il secondo pannello del dittico in cui Vagao mette in scena Judit che mette in scena se stessa.

Oloferne, spettatore privilegiato di entrambe le rappresentazioni, crede di possedere l'immagine di Judit e, in proiezione, il suo corpo, mentre in realtà ne è posseduto. Dapprima sembra che sia Vagao (inconsapevole strumento divino) a gestire le passioni del capo assiro. È però Judit che, attraverso un uso cosciente della propria immagine, domina Vagao (e, tramite lui, Oloferne). I due si servono l'uno dell'altra, in una sorta di lotta sotterranea per il controllo del comandante, il cui sguardo è presupposto sin dall'inizio del dialogo e che viene stregato dall'immagine "dipinta" dalla parola poetica. L'effetto di seduzione esercitato dal drammaturgo è tuttavia diverso da quello operato da Vagao su Oloferne: lo spettatore può infatti comprendere e ammirare non solo la bellezza dell'eroina, ma anche, e soprattutto, la sua potenza creativa nel soggiogare l'animo dei nemici. La rappresentazione della toletta di Judit si presta quindi a essere letta come teatralizzazione dell'intento di controllare le facoltà cognitive e immaginative dell'interlocutore tramite gli strumenti della parola e della visione.

Nell'iniziare la sua descrizione ammaliante, Vagao riconosce implicitamente la superiorità di Judit, confessando la vittoria che la prigioniera ha riportato su di lui, mezzano intrigante, grazie a un abile utilizzo sia della propria immagine sia del proprio eloquio. Vagao premette, infatti, al proprio racconto la sintesi dei pregi di Judit:

Che dirò, mio signor? Tutta è vaghezza, e se 'n volto è dea bella,

è dea anco in favella.

Il fascino di Judit non si limita all'aspetto, ma coinvolge la sua "favella", che sarà, accanto alla "vaghezza", la sua seconda ed altrettanto esiziale arma nello sconfiggere gli Assiri. Come Oloferne ammira l'abilità retorica 
di Vagao senza comprenderne l'intenzione manipolatoria, allo stesso modo il servo assiro, pur cogliendo la peculiarità di Judit, non è capace di intuirne la pericolosità. Accecato da un pregiudizio misogino, Vagao non riconosce nella "vaga favella" della sua interlocutrice la stessa arma manipolatrice di cui egli si serve nei confronti del proprio signore.

Nel costruire il proprio quadro, il servo dedica ampio spazio all'evocazione degli apparati fastosi che fanno da sfondo alla toletta della bella ebrea (cfr. vv. 1423-1440). L'ambientazione tutta interna della scena di denudamento, in contrasto con le quinte naturali di scene simili nella poesia pastorale (si pensi ad esempio all' Aminta del Tasso [II, 1]), sottolinea nel testo la collocazione cortigiana e, dunque, la valenza politica dell'episodio e del dramma. Resta tuttavia intatta la sensualità dell'episodio, che viene anzi accentuata sia dalla tensione tra l'artificio degli arredi e la nudità dell'eroina sia dal dettaglio del servo che spia, circostanza tipica degli intrighi cortigiani ${ }^{19}$.

In questo tableau vivant, di cui Vagao è l'unico regista, Judit non sa dello sguardo del servo e lo scoprirsi di lei ignara contribuisce notevolmente alla valenza erotica della scena, ricca di suggestioni dinamiche e di serici bagliori. L'immagine dell'eroina colta in un momento di abbandono viene, però, trasformata dall'intervento di Abra che, scoprendo il mezzano intento a spiare, induce la consapevolezza nei gesti di Judit.

Se prima si faceva ombra della propria clandestinità, Vagao adesso si fa impudente, chiedendo che Judit si presenti a lui così come si trova, adducendo come pretesto il messaggio di Oloferne. L'eroina accetta la sfida e si mostra in tutto il suo splendore. La sua bellezza e il suo fascino sono le armi con cui assume il controllo della situazione fino a capovolgerla, facendo del voyeur un proprio strumento tramite il quale inebriare e confondere Oloferne:

Così entrata è a chiamarla, et ella, uscita parte discinta e sciolta, parte ristretta e avolta, mentre or s'apre, or si copre, mille vaghezze scopre $[\ldots]$.

L'avvenenza di Judit è qui arricchita dalla presunta spontaneità del suo

${ }^{19}$ La suggestione lasciva della scena è stata segnalata in particolar modo da Giovanni Getto ("Il teatro barocco di Federico Della Valle", 1496-97) e da Franco Croce (Federico Della Valle, 146-148), che sottolinea anche l'ambientazione cortigiana dell'episodio e la conseguente valenza politica del giudizio etico. 
gestire. Siamo al momento di massima sensualità della scena: è difficile distinguere che cosa è natura e cosa è arte nell' incedere di Judit. Còlta di sorpresa "discinta e sciolta", ella accenna a coprirsi e il suo pudore (forse simulato nel concedere allo spettatore fuggevoli squarci della propria bellezza), rende ciò che si intravede ancora più desiderabile: sembra di assistere all'amplificazione degli effetti di sprezzatura citati dal Conte Ludovico di Canossa nel primo libro del Cortegiano ${ }^{20}$. La sensualità della figura di Judit al suo apparire di fronte al servo deriva, infatti, dall'assenza di artificio, o quantomeno dal ricorso a un artificio non immediatamente percettibile.

In questo primo quadro, controllato da Vagao, non si avverte ancora intenzionalità nello scoprirsi di Judit e l'eroina arriverà presto a teorizzare la naturalezza come propria inalienabile caratteristica. Bisogna tuttavia prestar fede solo in parte a tale strategia di autorappresentazione: nel momento stesso in cui rifiuterà ogni artificio assiro, la bella ebrea si costruirà davanti agli occhi del servo come un'opera d'arte. Proprio il ricorso dissimulato all'arte renderà Judit l'incarnazione iperbolica della femminilità castiglionesca. La natura proteiforme del suo personaggio viene sottolineata dall'adesione ad un modello riconosciuto, che tuttavia non dà interamente ragione della sua personalità poliedrica.

La lotta sotterranea fra Judit e Vagao è ormai serrata: compiacendosi del proprio potere, l'inviato di Oloferne ammira la prigioniera che si presenta a lui semisvestita e le offre le ricche gemme degli Assiri perché si orni adeguatamente, giustificando così l'arbitraria convocazione. Con apparente umiltà Judit si mostra a Vagao e ne rifiuta i gioielli, in nome di una presunta rivendicazione di semplicità. In realtà si assiste qui a uno scontro a vari livelli, tutti ugualmente importanti in un testo denso di valenze religiose e politiche:

20"Quanto più di tutte piace una, dico, non brutta, che si conosca chiaramente non aver cosa alcuna in su la faccia, benché non sia così bianca né così rossa, ma col suo color nativo pallidetta e talor per vergogna o per altro accidente tinta d'un ingenuo rossore, coi capelli a caso inornati e mal composti e coi gesti semplici e naturali, senza mostrar industria né studio d'esser bella? Questa è quella sprezzata purità gratissima agli occhi ed agli animi umani, i quali sempre temono essere dall'arte ingannati" Castiglione, Il Cortegiano, Libro I, xl, 88. Nella descrizione della Judit Della Valle sembra aderire alle teorie di Castiglione, solo che qui l'eroina non mostra una mano o un piede, ma tutte le sue grazie segrete. L'ambiguo erotismo di tali esempi di dissimulazione muliebre dell'artificio è discusso da Giulio Ferroni nel suo "Sprezzatura e Simulazione", dove nota come la sprezzatura costituisca un continuo atto di dissimulazione, se non di simulazione, reso necessario dalla catena di deviazioni e inganni che costituisce il comportamento sociale sulla "scena della corte" . 
— Giungerò serva indegna; - ha rispost'ella

[...]

A me fie assai ne l'abito, in cui prima

piacque al ciel ch'io piacessi,

non so se agli occhi o al core

del mio eccelso signore,

tornar a lui; ei mi riveggia quale

mi vide, ned io debbo, anzi non voglio

di nova o règia altezza

ornarmi et arrischiarmi

a dispiacergli, che mia morte fôra,

come 'l piacergli è cara

mia vita e mia grandezza. -

(vv. 1524; 1536-1546)

Judit è ben consapevole di come Vagao stia giocando col nuovo potere che gli proviene dal ruolo di mezzano. Nell'atto di mostrarsi remissiva, la donna passa in realtà al contrattacco, usando la propria bellezza come strumento di seduzione. Il rifiuto delle gemme assire è per l'eroina un modo per conservare la propria autonomia, ma è soprattutto un rifiuto della logica degli avversari: l'offerta dei gioielli vuole costringerla in un ruolo passivo, farne un oggetto decorativo, di cui i creatori possono disporre a loro piacimento. Ma l'avvenenza è per Judit un'arma segreta e personale, con la quale combatte in nome della sua gente; malgrado la protesta di semplicità, ella si abbiglierà in maniera sfarzosa, mostrandosi più affascinante di quanto i suoi interlocutori riuscissero a immaginarla, ma con le vesti fastose della propria famiglia, stirpe di guerrieri21. Il rifiuto di ornarsi "di nova o règia altezza" vale come ulteriore prova di sottigliezza nella risposta di Judit.

L'eroina persegue, infatti, i propri scopi senza mentire. Le basta tacere, senza dover accettare, ad esempio, il fine che implicitamente le attribuisce Vagao: l'ascesa sociale da umile prigioniera a favorita di Oloferne. Judit non simula, ma dissimula "onestamente": risponde il vero, ma mai in modo da consentire agli avversari di ricostruire il non detto e i suoi reali

${ }^{21}$ Il conflitto militare adombrato nel serrato confrontarsi di Judit e Vagao fa risuonare nel rifuto delle gemme assire, e dunque delle armi degli avversari, l'insistente richiamo di Machiavelli al ricorso ad "armi proprie" (cfr. fra tutti, Il Principe, cap. XII). L'eroina ebrea vince la sua battaglia con le proprie armi, rimanendo fedele a se stessa e alla sua causa fino alla fine. L'unità di propositi della protagonista si contrappone anche alla parziale infedeltà dei soldati assiri, che abbandoneranno i loro doveri di sorveglianza per dissetarsi alle fonti di Betulia, incoraggiati dal lassismo dei propri capi. 
propositi, comportandosi quindi come un'incarnazione ante litteram della dissimulazione onesta propugnata da Torquato Accetto. Tutte le sue parole suggeriscono un'attesa fervida, ma pudica, della notte in arrivo: possono, però, significare al tempo stesso sia compiacenza per i desideri di Oloferne, sia ansia di compiere la propria sacra e feroce missione ${ }^{22}$.

Nel non volersi rivestire di "nova" altezza, inoltre, Judit si oppone in maniera sottile sia al contegno di Vagao, che si esalta del proprio ruolo di mezzano del suo signore, sia a quello dello stesso Oloferne, sicuro di aver già vinto Betulia e convinto di essere un dio ${ }^{23}$. È come se l'ebrea volesse dichiaratamente prendere le distanze dalla hybris colpevole degli Assiri. A questo punto la protagonista passa alla controffensiva, invitando Vagao ad assistere a quel che resta della sua toletta: l'eroina comincia a modulare la recezione della propria immagine, assumendo il controllo, indirettamente, anche del resoconto di Vagao, a cui non resta che testimoniarne con stupore l'avvenenza sempre crescente.

Questa scena costituisce il secondo pannello del dittico (vv. 15591605 ) interamente dedicato al trionfo di Judit, che con ogni gesto soggioga Vagao e, tramite lui, Oloferne. A confermare questo capovolgimento dei rapporti di forza è il sorriso con cui la bella ebrea invita il mezzano a restare, elargito con la calma di chi sta giocando consapevolmente le sue carte ("e volea uscir. Ma ella, / placida quanto bella, / la dolce bocca ripiegando in riso, / ha detto: - Anzi ti ferma", vv. 1559 - 1562). Davanti al servo di Oloferne, e quindi per suo tramite nell'immaginazione del duce assiro, ella non a caso si riveste. Al campo semantico dello sciogliersi e dello scivolare, che caratterizzava la prima parte della toletta di Judit, si sostituisce quello del chiudere e dell'intrecciare, emblemi del recuperato controllo ( "Né s'è lavata sol, ma l'auree chiome / ha raccolte, ha intrecciate, me presente, / ha sparse, ha coronate / di ricche gemme, quali / ornan teste reali", vv. 1574-1578).

Judit costruisce se stessa come opera d'arte, orientando tramite una "divina favella" la percezione visiva della propria bellezza. Vagao, poeta-pittore abilissimo, non è, tuttavia, in grado di leggere il gioco simulatorio

${ }^{22}$ Per un'analisi del gioco semantico imbastito nel colloquio fra Judit e Oloferne, cfr. Raffaelli, Semantica tragica, 95.

${ }^{23}$ Si veda, fra i molti possibili esempi, la descrizione che ne dà Vagao ai vv. 708709 ("ma un dio vivo, splendente / d'arme pregne di lampi"), contrapponendo il feroce splendore del proprio comandante al deus absconditus dell'eroina. Immagine non meno icastica del blasfemo materialismo assiro è la grottesca minaccia di Oloferne al dio di Judit: "Ma s'io fo balenar questa mia spada, / ben so, ben so!" (vv. 514-515). 
della donna; è vittima dell'abilità di lei, che diviene la reale organizzatrice della scena. L'eroina finisce col gestire il gioco, sovvertendo dall'interno le regole imposte dagli avversari.

Vagao è preso al laccio: la sfrontatezza di chi ha violato con lo sguardo l'intimità della donna prigioniera, per poi chiamarla, discinta, dinanzi a sé, è sostituita dalla presunzione di essere stato testimone dell'epifania di una dea:

\section{[...] E'nver reina}

sembra propria d'Assiri,

s'a la pompa rimiri: ma nel volto

non è assiria né ebrea,

e poco è dirla dea. (vv. 1601-1605)

Il voyeur cortigiano diviene il portavoce del trionfo di Judit: del suo fascino certo, ma, inconsapevolmente, anche della sua abilità di maga che sconvolge a suo piacere la mente degli uomini. Leroina ebrea è una novella Armida, che per una volta ha snudato le proprie armi a favore del popolo eletto, dei predecessori di quei cristiani che si faranno carico della nobile missione della crociata contro i nuovi invasori orientali.

La scena dell'abluzione costituisce il momento chiave di questa dissimulata esibizione. L'eroina mostra al mezzano l'origine naturale del proprio fascino, in un passo di ricercata fattura:
Poi, vagamente assisa,
le man bianche e la fronte
s'è lavata soave; e sua bellezza,
non so come, lavando
crescer mi parea, in guisa
che s'accresce figura,
a cui giungendo vada arte e colore
curioso pittore.
(vv. 1566-1573)

L'acqua, elemento solvente e detergente, intensifica lo splendore della donna, che si avvolge di un alone di mistero. Il paragone tra Judit ed un pittore si carica, però, di ulteriori risonanze: nell'esaltare la purezza del proprio volto, infatti, la donna assicura che "Nulla cosa il pinge, / se non il sangue e Dio" (vv. 1564-1565), riprendendo il topos che oppone "natura" a "cosmesi" nella dialettica tra bellezza e seduzione. L'analogia con la pittura viene introdotta dall'eroina nel momento in cui assume un ruolo attivo nel definire la propria immagine. Vagao, in veste di poeta, sottolinea gli effetti del rituale di Judit: la bellezza della donna sembra "crescer" al contatto con l'acqua, così come si "accresce" l'immagine creata da un pittore 
nel passare da disegno a pittura. Judit raffina la propria bellezza servendosi di mezzi puri e legittimi. I suoi strumenti e i suoi colori sono di Dio e del sangue (la natura o forse la stirpe, in un gioco di dissimulazione onesta) oppure lei non è che il pennello, lo strumento di Dio e del "sangue", con riferimento metatestuale al topos del Deus pictor. L'eroina sembra attingere a questo tema, caro alla trattatistica classica e medievale, per sottolineare, con un'allusione che Vagao non può cogliere, l'origine provvidenziale delle proprie grazie 24 .

Alla naturalezza di Judit, còlıa mentre si lava, si oppone l'artificio della pittura, che si avvale dell'"aggiungere", non del "sottrarre" operato dall'acqua, elemento solvente. La corrispondenza, nel testo, fra naturalezza/sottrarre da un lato e artificio(arte)/aggiungere dall'altro ci viene confermata dalla giustapposizione delle due componenti del dittico. Nel primo quadro, infatti, la protagonista, ignara dello sguardo del servo, si spoglia, divenendo emblema della naturalezza che seduce nel suo manifestarsi, mentre nel secondo, tracciato consapevolmente da Judit, l'eroina si riveste, arricchendo il proprio fascino tramite l'artificio. La contrapposizione tra aggiungere e sottrarre, in cui il valore esplicitamente positivo è assegnato al secondo elemento, sembra inoltre riproporre e sintetizzare la differenza tra l'agire simulatorio di Vagao e quello dissimulante di Judit.

L'ambiguità che caratterizza il comportamento della bella ebrea sin dall'originale biblico rende necessaria la distinzione tra la condotta del mezzano e quella della bella prigioniera ${ }^{25}$. Se, infatti, la rhesis di Vagao amplifica la messa in scena dell'uso strumentale della retorica e delle arti visive, costruendo una rappresentazione della lotta controriformistica per il controllo delle coscienze, tale figurazione si colloca all'interno di un discorso di carattere etico sul mondo, come illustrano i commenti del coro. Sia il servo corrotto sia l'eroina (figura della Vergine) ricorrono in modo funzionale all'abilità oratoria e alla potenza dell'immagine. La valutazione morale degli strumenti della persuasione, d'altronde, sembra differire a seconda di chi se ne avvalga, tanto da far pensare ad una valutazione dei mezzi basata unicamente sulla legittimità dei fini.

${ }^{24}$ Sul topos del Deus pictor si veda Curtius, European Literature and the Latin Middle Ages, 544-546.

${ }^{25}$ Sulle molteplici suggestioni interpretative sollecitate dalla figura di Judit già nel testo biblico, si veda Squarzina, "Una, due, tre, cento Giuditte," nonché l'esaustiva indagine di Stocker, Judit Sexual Warrior. Stimolanti osservazioni sulle differenze tra l'originale biblico e la riscrittura dellavalliana sono sviluppate, oltre che nell'intervento di Squarzina, in Cazzani, "La vita e l'opera poetica di Federico della Valle"; Croce, Federico Della Valle, 126-128 e Gardair, "Giuditta e i suoi doppi," 457-463. 
Il testo mostra, tuttavia, come il comportamento di Vagao e quello di Judit non siano sovrapponibili. La diversa statura morale dei due antagonisti si associa, infatti, a due differenti strategie di comportamento. Vagao mente, si mostra servile e adulatore nei confronti di Oloferne e, se necessario, nei confronti di Judit, sebbene manipoli l'uno per brama di potere e disprezzi l'altra come prigioniera di guerra. Judit, come si è visto, ricorre invece alla dissimulazione onesta, tace i suoi fini, ma non sconfessa i suoi valori. È in grado di leggere il mondo e di non farsi guardare nel cuore, pur senza mentire.

Con questa creazione, Federico della Valle legittima, dunque, l'uso difensivo delle tecniche della persuasione, entro una visione complessivamente pessimistica del mondo e del potere ${ }^{26}$. Nella prospettiva desolata messa in scena nelle sue tragedie, nessun potere terreno può attingere alla giustizia. Solo ai subordinati e agli umili è dato seguire la volontà di Dio. In un mondo regolato da leggi violente, che comportano il disconoscimento della virtù e il controllo oppressivo del pensiero e della libertà di coscienza, la dissimulazione è uno strumento fondamentale affinché possa realizzarsi sulla terra il progetto di Dio sull'uomo. Le figure femminili, in particolar modo, sembrano le depositarie privilegiate della misteriosa e imperscrutabile azione divina, proprio grazie alla posizione marginale in cui vorrebbe confinarle lo sguardo maschile. La cecità dei loro interlocutori, che non si curano di indagarne i pensieri segreti, permette, così, alle eroine dellavalliane di intervenire con particolare incisività nel dramma della storia.

\section{Rutgers University}

\section{Opere citate}

Accetto, Torquato. Della dissimulazione onesta. A cura di Salvatore Nigro. Torino: Einaudi, 1997.

Angelini, Franca. Il teatro barocco. Bari: Laterza, 1975.

Benson, Pamela J. The Invention of the Renaissance Woman. The Challenge of Female Independence in the Literature and Thought of Italy and England. University Park, PA: Pennsylvania State UP, 1992.

Castiglione, Baldassar. Il Cortegiano. Introduzione di Amedeo Quondam, note di

Nicola Longo. Milano: Garzanti, 2000.

Cazzani, Pietro. "La vita e l'opera poetica di Federico della Valle" pp. vii-liii in Federico Della Valle. Tutte le opere. Milano: Mondadori, 1955.

26 L'universalità del pessimismo dellavalliano è registrata in numerosi interventi critici, tra i quali segnaliamo Trombatore, "Le tragedie di Federico Della Valle"; Croce, Federico Della Valle, e "Federico della Valle". 
Chemello, Adriana. "Donna di palazzo, moglie, cortigiana: ruoli e funzioni sociali della donna in alcuni trattati del Cinquecento" pp. 113-132 in La Corte e il "Cortigiano. II. Un modello europeo. A cura di Adriano Prosperi. Roma: Bulzoni 1980.

Ciletti, Elena. "Patriarchal Ideology in the Renaissance Iconography of Judith" Pp. 35-70 in Refiguring Woman. Perspectives on Gender and the Italian Renaissance. A cura di Marylyn Migiel e Juliana Schiesari. ithaca: Cornell Univesity Press, 1991.

Croce, Franco. Federico Della Valle. Firenze: La Nuova Italia, 1965.

Curtius, Ernst Robert. European Literature and the Latin Middle Ages. Princeton: Princeton University Press, 1967.

Daenens, Francine. "Superiore perché inferiore: il paradosso della superiorità della donna in alcuni trattati del Cinquecento" pp. 11-50 in Trasgressione tragica e norma domestica. Esemplari di tipologie femminili dalla letteratura europea. A cura di Vanna Gentili. Roma: Storia e Letteratura, 1983.

Della Valle, Federico. Tragedie. A cura di Andrea Gareffi. Milano: Mursia, 1988.

Ferroni, Giulio. "Sprezzatura e simulazione" pp. 119-147 in La Corte e il Cortegiano. I La scena del testo. A cura di Carlo Ossola. Roma: Bulzoni, 1980.

Gardair, Jean-Michel. "Giuditta e i suoi doppi" pp. 457-463 in I Gesuiti e i primordi del teatro baroco in Europa. Convegno di studi. A cura di Maria Chiabò e Federico Doglio. Roma: Centro studi sul teatro medioevale e rinascimentale, 1994.

Getto, Giovani. "Il teatro barocco di Federico Della Valle" vol. 2, pp. 1489-1521 in Letteratura Italiana. I Minori. 5 voll. Milano: Marzorati, 1961.

"Federico della Valle", pp. 253-255 in Dizionario enciclopedico della letteratura italiana. Bari: Laterza, 1966.

Fumaroli, Marc. Eroi e oratori. Retorica e drammaturgia secentesche. Bologna: Il Mulino, 1990.

Kelly, Joan. "Did Women Have a Renaissance?" pp. 19-50 in Women, History, and Theory: The Essays of Joan Kelly. Chicago and London: Chicago University Press, 1984.

King, Margaret L. Women of the Renaissance. Chicago: Chicago University Press, 1991.

Mercuri, Roberto. "La Reina di Scozia di Federico Della Valle e la forma della tragedia gesuitica" Calibano, 4 (1979): 142-161.

La Penna, Antonio. "Palazzo, coro e popolo nella tragedia antica e nella tragedia umanistica” pp. 79-111 in Mito, realtà e potere nel teatro: dall'antichità classica al Rinascimento. A cura di Maria Chiabò e Federico Doglio. Viterbo: Centro studi sul teatro medioevale e rinascimentale, 1987.

Paratore, Ettore. "Nuove prospettive sull'influsso del teatro classico nel ' 500 " pp. 9-95 in Atti del Convegno sul tema Il teatro classico italiano nel '500. A cura di Enrico Cerulli et al. Roma: Accademia Nazionale dei Lincei, 1971.

Pietropaolo, Domenico. "Iudit, Femme Fatale of the Baroque Stage" pp. 273-283 in Donna. Women in Italian Culture. A cura di Ada Testaferri. Ottawa: Dovehouse, 1989. 
Raffaelli, Sergio. Semantica tragica di Federico Della Valle. Padova: Liviana, 1973. Sanguineti White, Laura. Dal detto alla figura. Le tragedie di Federico Della Valle. Firenze: Leo S. Olschki, 1992.

Squarzina, Luigi. "Una, due, tre, cento Giuditte", Lettere Italiane, 51, 1 (1999): 52-69.

Stocker, Margarita. Judit Sexual Warrior. Women and Power in Western Culture. New Haven and London: Yale University Press, 1998.

Tessari, Roberto. "La Iudit di Della Valle: Pitture di sperati diletti" pp. 109-124 in

Teatri Barocchi. Tragedie, commedie, pastorali nella drammaturgia europea fra '500 e '600. A cura di Silvia Carandini. Roma: Bulzoni, 2000.

Trombatore, Gaetano. "Le tragedie di Federico Della Valle" pp. 165-192 nel suo Saggi critici. Firenze: La Nuova Italia, 1950. 